\title{
Programación e implementación del software de aplicación "Fácil Hogar" para aminorar el índice de desperdicio de alimentos en los hogares de Tlaxcala
}

\author{
Amado Sánchez, Josefina Angelino, Daniel Temoltzin, Arturo Águila \\ Universidad Politécnica de Tlaxcala, Hueyotlipan, \\ Tlaxcala, México \\ \{amado.sanchez1982, josefinaaz12\}@gmail.com, \\ \{dtvega2010, ing.arturo_aguila\}@ hotmail.com
}

\begin{abstract}
Resumen. Datos del Banco Mundial reflejan que entre un cuarto y un tercio de los alimentos producidos anualmente para consumo humano a nivel mundial se desperdician; en México se pierden 10 millones de toneladas de alimentos por caducidad. Las principales causas provienen del comportamiento del consumidor teniendo un elevado índice de pérdidas en el hogar equivalente a un $28 \%$. Se presenta el diseño del Software de aplicación "Fácil Hogar" dirigido a amas de casa de zonas urbanas en Tlaxcala, creado en la plataforma APP INVENTOR 2, que permite la administración de entradas y salidas de los alimentos adquiridos. El presente proyecto propone contrarrestar los desperdicios generados en el hogar, controlando las entradas y salidas de la alacena y el refrigerador, indicándole a la ama de casa el estado del alimento en base a su fecha de caducidad para que sepa en todo momento que productos debe consumir en primera instancia. Dentro de los resultados alcanzados se obtuvo una mejora en disminuir el desperdicio de alimentos y costos en el hogar.
\end{abstract}

Palabras clave: Aplicación, App Inventor, alimentos, desperdicio, reducción.

\section{Programming and Implementation of the "Easy Home" Application Software to Reduce the Food Waste Index in Tlaxcala Households}

\begin{abstract}
World Bank data show that between one-quarter and one-third of the food produced for human consumption worldwide is wasted annually; in Mexico 10 mil-lion tons of food per expiry are lost. The main causes are consumer behavior, with a high loss rate in the household of $28 \%$. The design of the "Easy Home" application software for urban housewives in Tlaxcala, created in the APP INVENTOR 2 platform, which allows the administration of inputs and outputs of purchased foods, is presented. Counteract the waste generated in the home, controlling the entrances and exits of the cupboard and the refrigerator, indicating to the housewife the state of the food based on its expiration date so that it knows
\end{abstract}


at all times which products to consume in the first instance Within the results obtained, an improvement was obtained in reducing food waste and costs in the household.

Keywords: Application, App Inventor, food, waste, reduction.

\section{Introducción}

El objetivo del presente proyecto se basa en desarrollar una aplicación para dispositivos Android que permita a las amas de casa contrarrestar los desperdicios generados en el hogar, controlando las entradas y salidas de la alacena y el refrigerador, indicándole a la ama de casa el estado del alimento en base a su fecha de caducidad para que sepa en todo momento que productos debe consumir en primera instancia.

Para llevar a cabo el desarrollo de la aplicación se debe tener en cuenta que dentro del mercado de dispositivos móviles, los que están experimentando un mayor crecimiento de ventas son los tablets y los smartphones. Estos últimos serán los utilizados para poder crear la aplicación.

Actualmente, alrededor del 53.4 millones de dispositivos móviles existen en México, lo que permite una mayor expansión en el uso de la aplicación desarrollada.

\subsection{Motivación del desarrollo de la aplicación}

Aproximadamente 870 millones de personas pasan hambre todos los días, mientras que la asombrosa cantidad desperdicio de alimentos en el mundo ha crecido exponencialmente. De acuerdo con la Organización de las Naciones Unidas para la Alimentación y la Agricultura 1,300 millones de toneladas anuales se pierden o desperdician a escala mundial. Generalmente se desperdicia el $50 \%$ de frutas y verduras, $30 \%$ de cereales, $60 \%$ de tubérculos, y $20 \%$ de alimentos de origen animal. FAO (2012). Se estima que el $6 \%$ de las pérdidas mundiales de alimentos se dan en América Latina y el Caribe. En América Latina se pierde el 15\% de los alimentos que se producen cada año, es decir 80 millones de toneladas, lo que significa que cada habitante desperdicia $220 \mathrm{~kg}$ de alimentos al año.

Con respecto a México el desperdicio es de más de 10 millones de toneladas de alimentos anuales, lo cual representa el $37 \%$ de la producción agropecuaria en el país. Las pérdidas de alimentos en México tienen lugar en las etapas de procesamiento $6 \%$, mercado $17 \%$, manejo y almacenamiento $22 \%$, producción $28 \%$ al igual que en hogares.

\subsection{Objetivo}

Desarrollar una aplicación dirigida a las amas de casa que le permita llevar un control de entradas y salidas de sus alimentos. 
Programación e implementación del software de aplicación "Fácil Hogar” para aminorar ...

\subsection{App Inventor 2}

App Inventor es una aplicación originalmente desarrollada por Google y mantenida ahora por el Instituto de Tecnología de Massachusetts. Permite que cualquier persona, incluyendo las no familiarizadas con la programación y SDK de Android, pueda crear aplicaciones de Software para Android. Utiliza una interfaz gráfica, muy similar al Scratch y el StarLogo, que permite a los usuarios arrastrar y soltar objetos visuales para crear una aplicación que puede ejecutarse en el sistema Android. Google puso fin al desarrollo el 31 de diciembre de 2011 cediéndole el código al MIT, quien lo ha puesto a disposición de todos. Se trata de una utilidad Web desarrollada por Google que permite realizar aplicaciones para Android sin escribir código, todo de forma visual e intuitiva (uniendo piezas de un puzle). Utiliza una interfaz gráfica que permite a los usuarios arrastrar y soltar objetos visuales para crear una aplicación que puede ejecutarse en el sistema Android que funciona en muchos dispositivos móviles. Todo ello sin usar ni una sola línea de código, de forma intuitiva y gráfica. Molina, (2006).

En la creación de App Inventor para Android, Google se basó en la investigación de la informática educativa, y el trabajo realizado en entornos de desarrollo en línea. El editor de bloques utiliza la biblioteca Open Blocks de Java para la creación de lenguajes de programación visuales. Open Blocks está distribuida por el Massachusetts Institute of Technology Program's Scheller para formación de profesorado y deriva de la investigación de la tesis de Ricarose Roque. El profesor Eric Klopfer y Daniel Wendel del Programa Scheller apoyaron la distribución de bloques abiertos bajo la licencia MIT. La programación de bloques abiertos y visuales está estrechamente relacionada con el StarLogo, un proyecto de Klopfer, y Scratch. Estos proyectos están formados por teorías del aprendizaje construccionista, que hace hincapié en que la programación puede ser un vehículo para conseguir ideas de gran alcance a través del aprendizaje activo. Como tal, es parte de un movimiento continuo en las computadoras y la educación que se inició con el trabajo de Seymour Papert y el Grupo de StarLogo del MIT en 1960, y también manifestado en el trabajo de Mitchel Resnick, Lego Mindstorms y StarLogo. El equipo de App Inventor fue dirigido por Hal Abelson y Mark Friedman.

\section{Diseño metodológico}

Esta investigación se realizó en dos fases:

- La estrategia metodológica (ver fig. 1), que implicó: el contexto, el marco teórico, el problema y los tipos de investigación, con base en ello se estableció el diseño y la segunda fase.

- El trabajo empírico, que consistió en la obtención, análisis y presentación de la aplicación. En esta etapa se incluye la metodología de desarrollo del software, así como el desarrollo de la interfaz. 


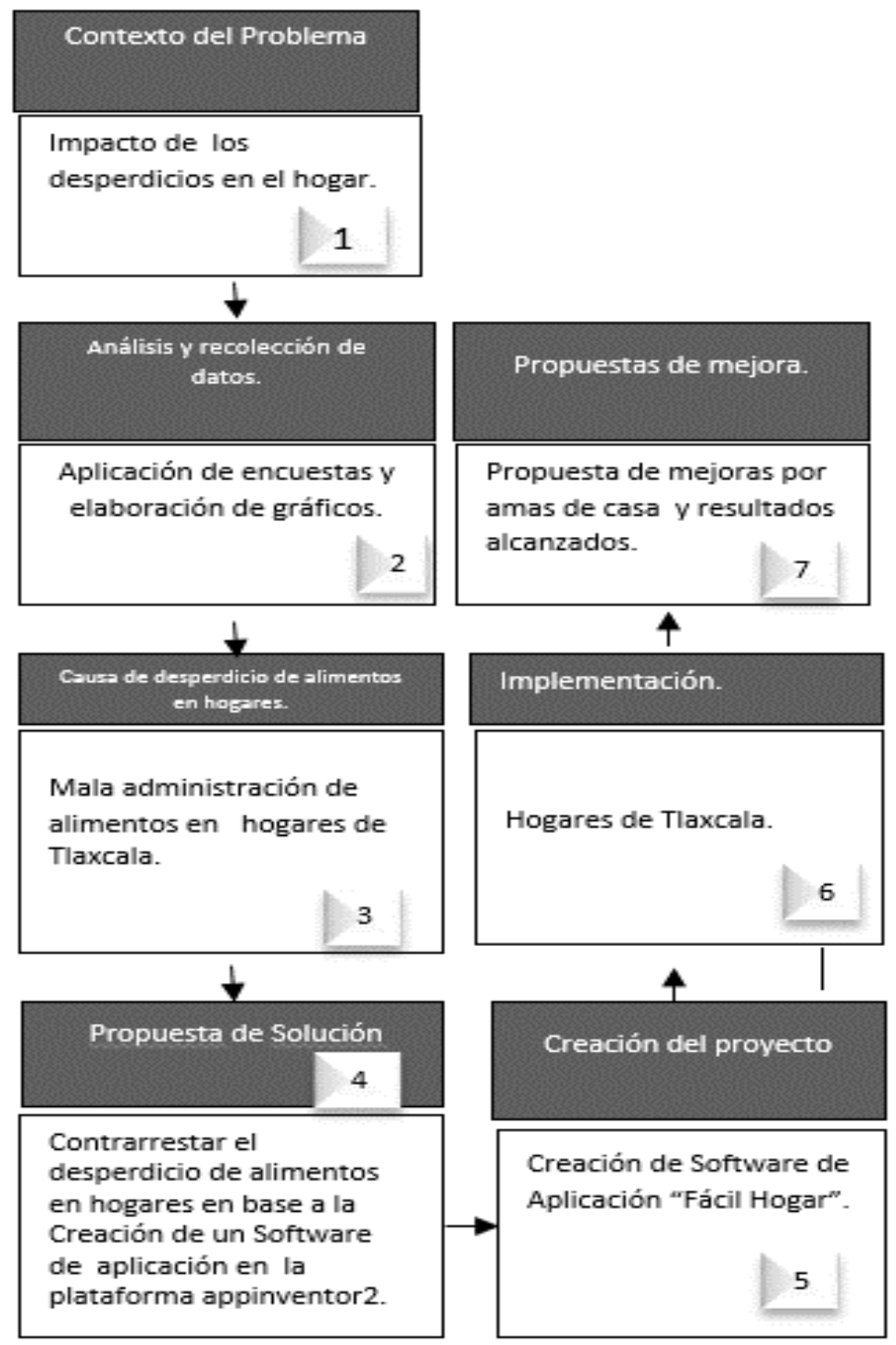

Fig. 1. Estratégia metodológica.

\subsection{Metodología de desarrollo de software}

La figura 2, muestra la metodología empleada para el desarrollo del software "fácil hogar" misma que fue selecciona luego de revisar las propuestas en JISBD (2003), Yusef (2004), Sommerville (2005) y Schwaber (2013), a fin de generar un producto de manera rápida y funcional. A su vez, tomando en consideración la investigación de Catalán V., se diseñó la interfaz de usuario en una primera etapa de tal forma que fuese amigable con el usuario, en este caso la ama de casa. 
Programación e implementación del software de aplicación “Fácil Hogar” para aminorar ...

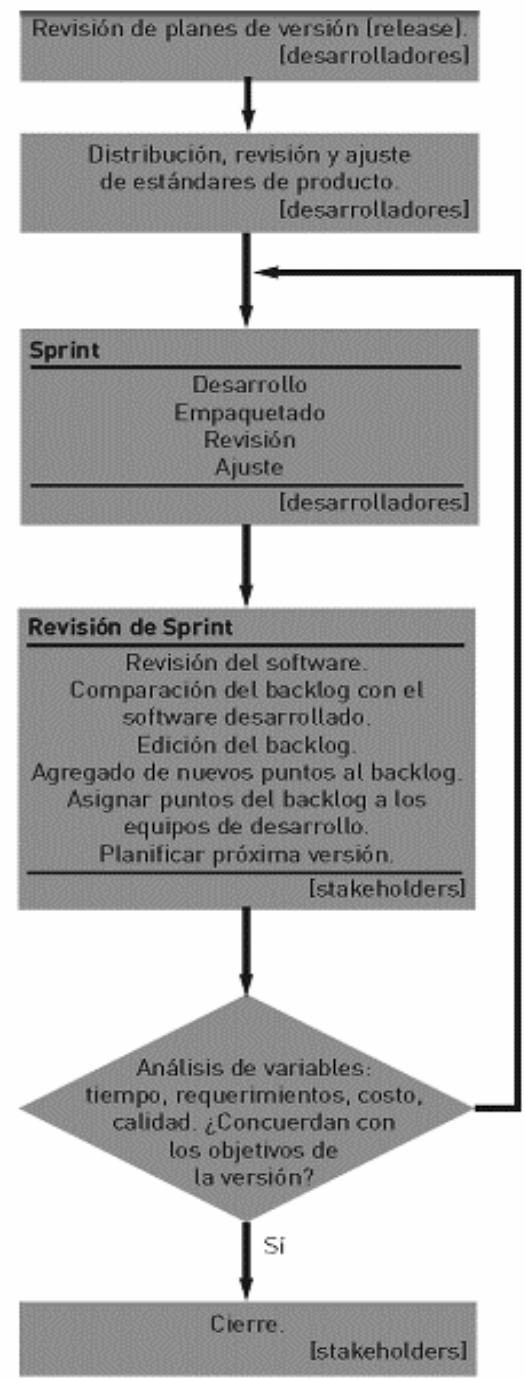

REVISIÓN DE PLANES DE VERSIÓN:

Se revisa que hay que hacer y en que punto está la distribución actual.

\section{SPRINT:}

Es la fase de desarrollo iterativa.

Desarrollo: Análisis, implementación, testing. Empaquetar: Generar paquetes ejecutables Revisión: Resolución de problemas y se añaden nuevos ítems.

Ajustes: Uso de los ajustes para mejorar el producto.

\section{SPRINT REVIEW:}

Después del Sprint se hace una reunión con el ScrumMaster donde se revisa el producto del Sprint anterior y en el que se pueden añadir puntos nuevos al backlog.

\section{Cierre:}

En esta fase se encuentran las típicas actividades de fin de proyecto como, hacer una versión distribuible, testear, marketing etc....

Fig. 2. Metodología Scrum para desarrollo de Software.

\section{Desarrollo}

\subsection{Contexto del Problema}

En 2007, se utilizaron casi 1.400 millones de hectáreas de tierras para producir alimentos que no se consumieron. Esto representa una superficie más grande que Canadá y la India juntos. El despilfarro de alimentos contribuye a la expansión agrícola hacia zonas silvestres lo que se traduce en pérdidas de especies, incluidos mamíferos, 
aves, peces y anfibios. Los alimentos se pierden o desperdician en toda la Cadena de Suministro, en México existe una mayor pérdida de alimentos en el sector de consumo es decir en los hogares, con una de pérdida del $28 \%$. Las causas de desperdicio de alimentos en los hogares se basan principalmente en aspectos relacionados con las fechas de consumo. McMahon (2011).

Esta misma problemática se ve reflejada en los hogares de la entidad de Tlaxcala que se han visto afectados por la pérdida de alimentos debido a la fecha de caducidad teniendo un impacto negativo en la economía de los hogares. La investigación se aplica en la ciudad de Apizaco Tlaxcala debido a que es una zona urbana y las amas de casa están familiarizadas con el uso de la tecnología.

\subsection{Análisis y recolección de datos}

Con el propósito de obtener la información sobre el desarrollo de la aplicación "fácil hogar" se llevó a cabo una encuesta de opinión. Tomando una muestra con un nivel de confianza de $95 \%$ y se aplicó la encuesta a 300 amas de casa. La encuesta consistió en una entrevista directa y personal, en las cuales se les cuestionaron los aspectos diversos sobre el desperdicio de alimentos como:

- ¿En qué basa el orden de consumo de sus alimentos?

- ¿Hace uso de las tecnologías (Tablet, Celular, Internet)?
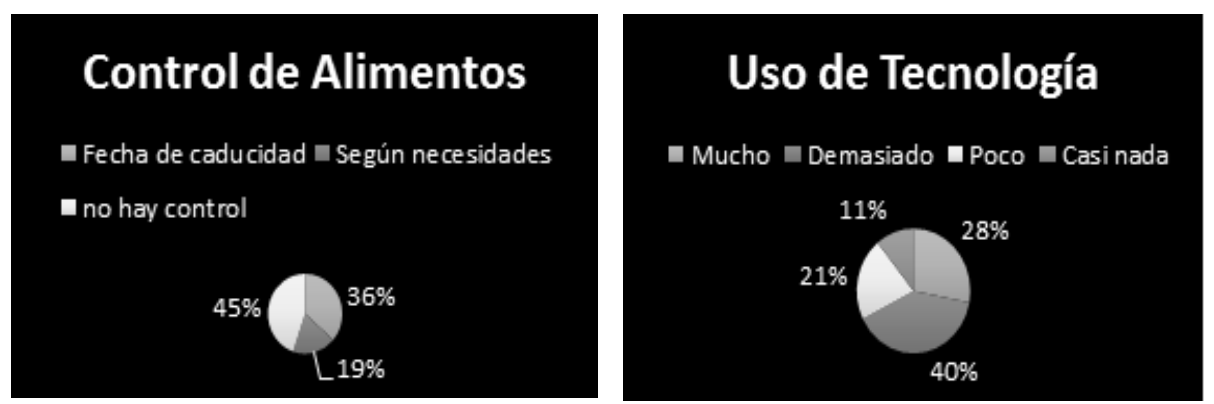

Fig. 3. Control de alimentos y uso de tecnología (creación propia).

Como resultado de la primera pregunta se obtuvo que el $45 \%$ de los encuestados no tiene un control sobre las entradas y salidas de sus productos lo que ocasiona una elevada cantidad de desperdicio y un incremento considerable en sus costos, mientras que el $36 \%$ no toma en cuenta las fechas de caducidad de los producto, y por último con un $19 \%$ las compras las realiza de acuerdo a sus necesidades. En la figura 3, se muestra los resultados obtenidos de la encuesta realizada a las amas de casa en cuanto al control de los alimentos y el uso de la tecnología. Respecto los resultados obtenidos con respecto a las amas de casa que utilizan tecnología (Tablet, celular, internet), el $40 \%$ de las amas de casa están en contacto directo con la tecnología, el 28\% muestra un uso frecuente con celular o tablet. Estos indicadores nos muestran que la aplicación a desarrollar será de uso frecuente para la administración de los alimentos. 


\subsection{Causa de desperdicio de alimentos en los hogares de Tlaxcala}

En base a los resultados obtenidos anteriormente se encontró que la causa principal de desperdicio en los hogares de Apizaco es que el ama de casa no tiene una correcta administración del consumo de sus alimentos, debido a que ignora la fecha de caducidad de los productos, consumiendo en primer lugar los que tienen una fecha de caducidad más alejada y dejando rezagar los próximos a caducar.

Después del análisis de la situación e identificación de las principales causas de desperdicio de alimentos se procede a la etapa 4, la propuesta de solución en la cual se propone diseñar y construir un Software de aplicación para los celulares o tabletas llamado "fácil hogar", el cual consiste en un conjunto de formularios que permite al ama de casa visualizar de manera rápida y fácil los alimentos que están más próximos a caducar los cuales deben ser consumidos en primer lugar, así como también le permite llevar un efectivo control de existencias, todo esto con la finalidad de contrarrestar el desperdicio de alimentos y optimizar los costos.

\subsection{Creación del software}

La creación del software de Aplicación se realizó en la plataforma de App Inventor2 en 5 fases, se describen como sigue:

1. Formulario de Inicio. Permite a la ama de casa ingresar a la aplicación por medio de reconocimiento de voz, utilizando el componente Speech Recognizer el cual permite reconocer patrones de voz y llevarlas a texto. En la figura 4 se muestra el formulario y los bloques utilizados para su programación.

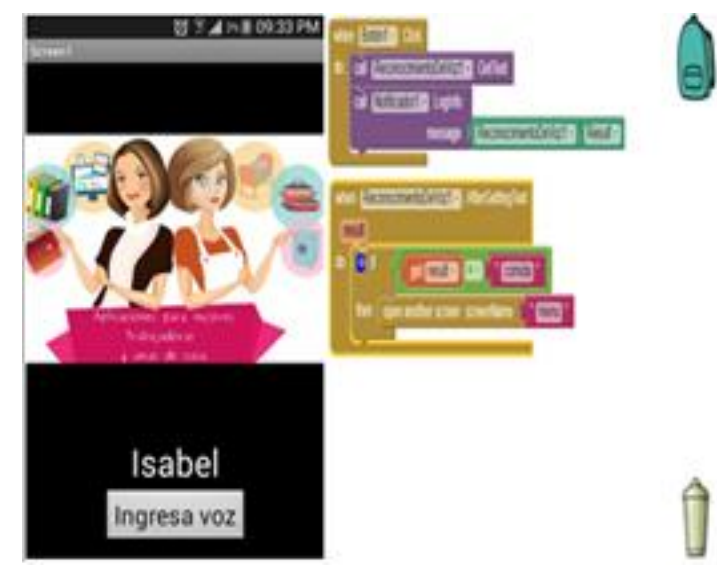

Fig. 4. Formulario de ingreso (creación propia).

2. Formulario de Menú. Le permite a la ama de casa ingresar a los diferentes formularios de la aplicación según sus necesidades, en este formulario se utiliza el componente Open Another Screen, este componente ayuda a la programación de los botones para crear un vínculo de un formulario a otro. En la gura 5 se muestra el formulario y sus bloques de programación correspondientes. 

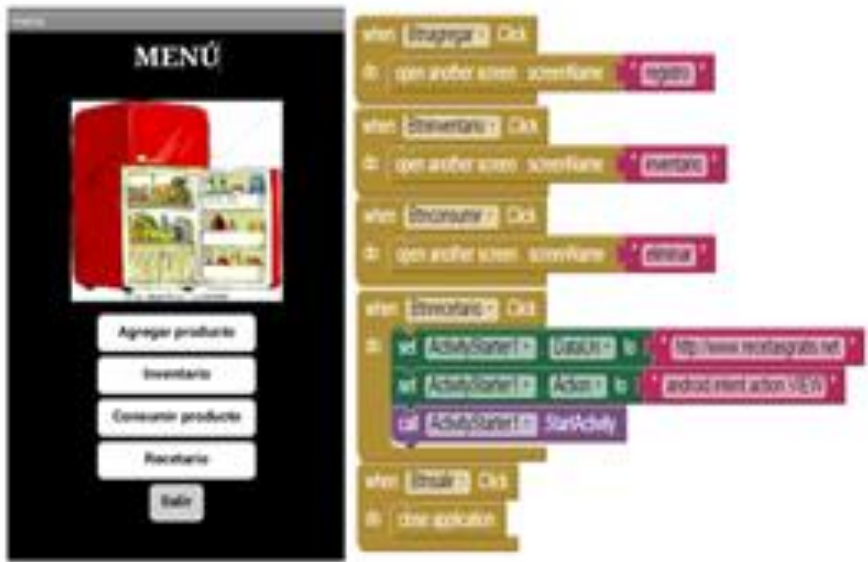

Fig. 5. Formulario del menú (creación propia).

3. Formulario de registro de producto. En este formulario la Ama de Casa podrá registrar sus productos del refrigerador o la alacena, para el funcionamiento correcto del registro se necesita descargar un lector de barras llamado Barcoder Scaner ya que es indispensable registrar este campo para que sirva como elemento de búsqueda en otros formularios, también se utiliza el componente Date Picker para ajustar la fecha actual, los datos que se ocupan son nombre del producto, fecha de caducidad, código de barras. En la gura 6 se muestra el formulario de registro de productos.

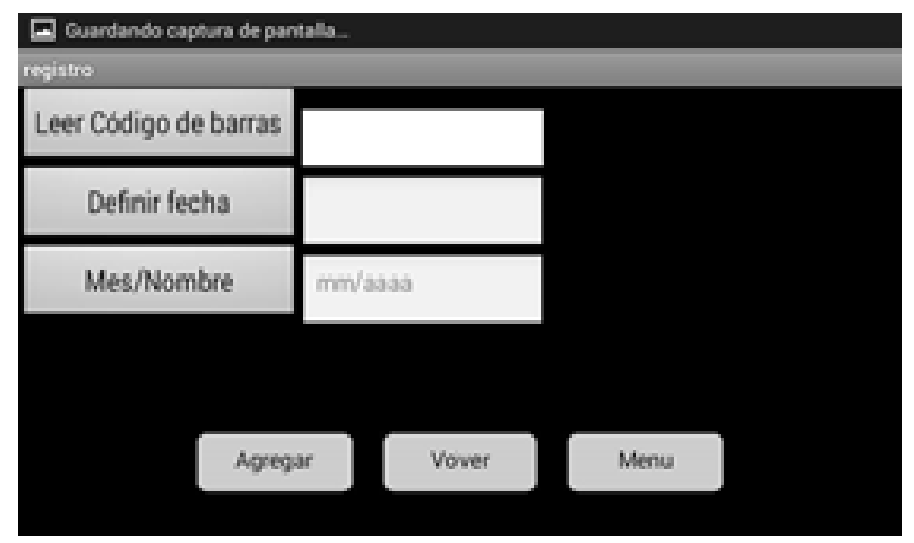

Fig. 6. Formulario de productos (creación propia).

4. Formulario de consumo. En este formulario el ama de casa podrá eliminar de su base de datos los productos que se han consumido, para programar esta aplicación se utilizaron los comandos llamados Delete que permiten eliminar de manera permanente los productos de la base de datos. Para poder elegir los productos almacenados en la memoria interna del dispositivo se requiere que el producto sea buscado por medio del 
código de barras ya que es un dato que no se repite en ningún producto. En la figura 7 se muestra el formulario y los bloques programados para el correcto funcionamiento del formulario.

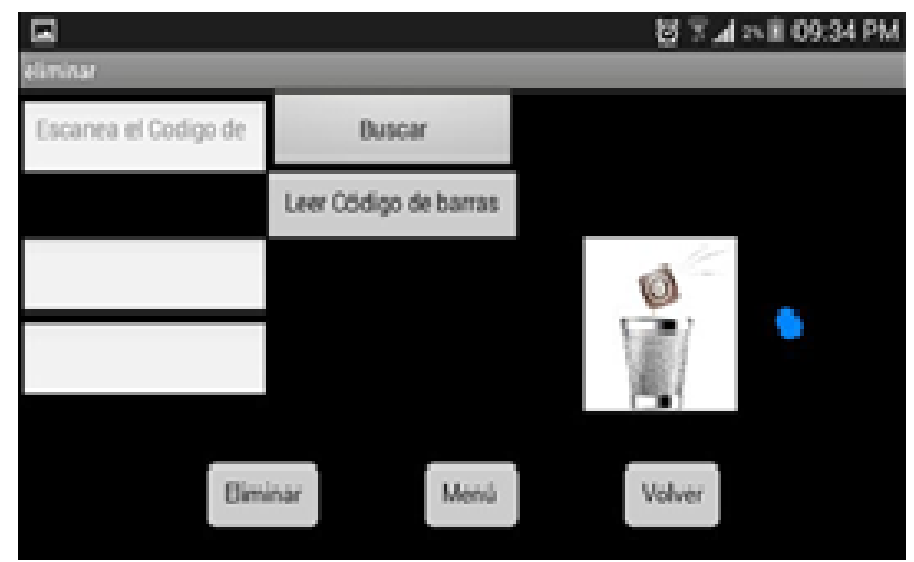

Fig. 7. Formulario de consumo (creación propia).

5. Formulario de Inventario. En este formulario el ama de casa puede visualizar la existencia de sus productos, los productos son almacenados en la memoria interna del dispositivo con la ayuda de la base de datos Tynni este elemento permite almacenar los datos en la memoria interna del dispositivo, este formulario contiene el elemento Show lterbar uno de los más importantes de la aplicación, en este se pueden filtrar los productos por nombre o por fecha de caducidad para conocer cuáles son los productos que se deben de consumir en primer lugar. En la Figura 8 se muestra el formulario y los bloques de programación delInventario.

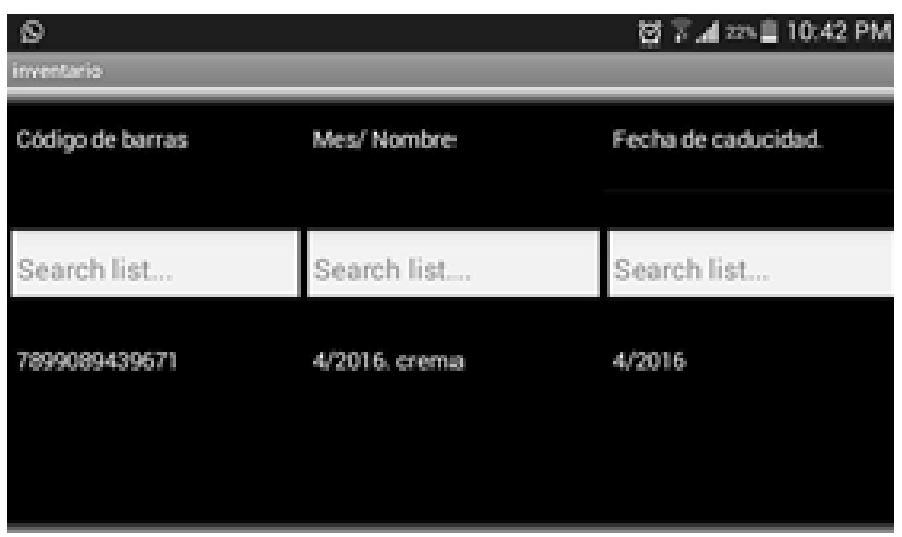

Fig. 8. Formulario de inventario (creación propia).

Debido a las características de la plataforma utilizada para la Creación de este Software de Aplicación, solo podrá funcionar si se cuenta con una conexión Wi-Fi. 


\section{Resultados obtenidos}

Para comprobar la funcionalidad del Software de Aplicación "fácil hogar, se procedió a la implementación" en 10 hogares de la ciudad de Apizaco Tlaxcala del 1 al 30 de Abril del 2016, teniendo una participación favorable de las Amas de casa, ya que estaban muy interesadas por aprender a usar la Aplicación obteniéndose los siguientes resultados.
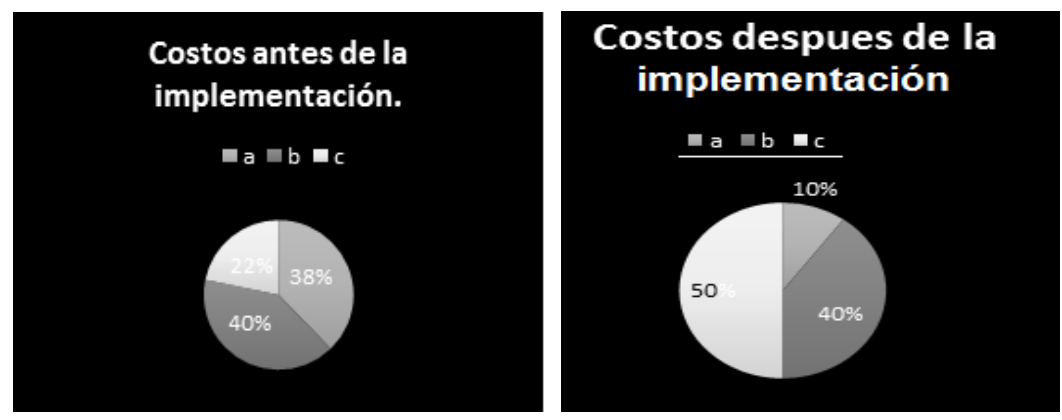

Fig. 9. Comparación de costos antes y después de la implementación.

En las gráficas de la figura 9, puede observarse la comparación de los costos de las amas de casa en alimentos antes de la implementación de la aplicación. El ama de casa gastaba entre \$2000 y \$3000 pesos y después del uso de la Aplicación gastó de \$800 a $\$ 1000$ pesos teniendo como resultado un ahorro de $\$ 1500$ pesos por hogar.

\section{Conclusiones}

El desperdicio de alimentos es mayor aún en un hogar, debido a que los alimentos que se tienen almacenados se ha dejado que se estropeen o caduquen por negligencia, se estima que el valor de los alimentos que se pierden o desperdician cada año en todo el mundo es de un billón de USD. Ahora bien las causas de desperdicio de alimentos en el hogar están principalmente relacionadas con el comportamiento del consumidor como planificar inadecuadamente las compras y no consumir los alimentos antes de su fecha de caducidad también conlleva un desperdicio de alimentos evitable. Es por ello la importancia de comprender primeras entradas, primeras salidas, considerando obtener reducción de costos, y desperdicios ya que las pérdidas de alimentos representan una pérdida del valor económico para los actores de las cadenas de producción y suministro de alimentos. En ultimátum recae en primeras entradas, primeras salidas, técnica de gestión que trata de distribuir los alimentos por la cadena de suministro seleccionando primero los que caduquen antes (First Expires) y, al igual de caducidad más antigua (First Out). Así la caducidad se convierte en el eje sobre el que gira todo el funcionamiento de un almacén. En este lapso de dominar el control de caducidades de alimentos con primeras entradas y primeras salidas, se incorporan diferentes formas para evitar desperdicios y reducir costos, como el apoyo de las 
Programación e implementación del software de aplicación “Fácil Hogar” para aminorar ...

tecnologías con uso de aplicaciones, en éste caso. Se ha determinado que implementando nuevas tecnologías al uso cotidiano de un hogar, puede apoyar en disminuir los índices de desperdicios y mejorar los niveles de alimentación. El ama de casa puede realizar sus labores domésticas de forma interactiva y controlada, con el Software de aplicación tendrá a su alcance los productos y alimentos comprados desde un supermercado y así facilitar su consumo. Este proyecto permitió estimular el enfoque educativo y alimenticio ya que su desarrollo se basó sobre la disminución de desperdicio en un hogar con un funcionamiento exitoso debido a que se ha disminuido considerablemente el desperdicio de alimentos además de que se observó una reducción en los gastos alimentarios de la muestra.

\section{Referencias}

1. INEGI: Estadísticas históricas de México. 8, Agropecuario, aprovechamiento forestal y pesca, México: INEGI (2009)

2. Molina, J., Córdova, L.: App Inventor Mit 2 (2006)

3. Censo Agropecuario: Panorama Agropecuario en México: Censo Agropecuario. Instituto Nacional de Estadística y Geografía, México, INEGI (2007)

4. McMahon, M., Valdés, A., Cahill, C., Jankowska, A.: Análisis del Extensionismo Agrícola en México, México, SAGARPA (2011)

5. FAO: Pérdidas y Desperdicio de Alimentos en el Mundo, Alcance, Causas y Prevención. Roma, FAO (2012)

6. Wyman, O.: Reducir el desperdicio de los alimentos, ¿Cómo pueden las empresas de distribución ayudar? Estados Unidos de Norte América (2014)

7. FAO: Iniciativa mundial sobre la reducción de la pérdida y el desperdicio de alimentos. Roma, FAO (2014)

8. HLPE: Las pérdidas y el desperdicio de alimentos en el contexto de sistemas alimentarios sostenibles. Un informe del Grupo de alto nivel de expertos en seguridad alimentaria y nutrición del Comité de Seguridad Alimentaria Mundial, Roma (2014)

9. Taller Metodologías Agiles en el Desarrollo de Software: Taller realizado en el marco de las VIII Jornadas de Ingeniería del Software y Bases de Datos, Universidad Politécnica de Valencia, JISBD (2003)

10. Hassan, Y., Martín, F. J., Iazza, G.: Diseño Web Centrado en el Usuario: Usabilidad y Arquitectura de la Información. Hipertext.net, No. 2, http://www.hipertext.net (2004)

11. Sommerville, I.: Ingeniería del Software. Séptima edición, Pearson (2005)

12. Schwaber, K., Sutherland, J.: La Guía de Scrum, Las Reglas del Juego (2013)

13. Catalán, V. M.: Metodología de evaluación de interfaces gráficas de usuario. http://eprints.rclis.org/6732/1/Metodologias_de_evaluaci\%C3\%B3n_de_interfaces_gr aficas_de_usuario.pdf 